\title{
Design and Simulation of 5-Bit DMTL Phase Shifter with Improved RF Switch Incorporation of Stress Regions
}

\author{
${ }^{1}$ G Srihari, ${ }^{\otimes 2}$ T. Shanmuganantham \\ ${ }_{1,2}$ Department of Electronics Engineering, Pondicherry University, Puducherry, India 605014 \\ srihari.nan@gmail.com, shanmugananthamster@gmail.com
}

Received: 01st July 2020, Accepted: 27th July 2020, Published: 31st August 2020

\begin{abstract}
The MEMS technology was the most adopted technology in recent years in the field of Communication which involves the RF devices. Rapid changes have been incorporated in passive component design with use Microsystems, having immense advantage over traditional with small size, better performance and compatibility with limited energy usage. Among, Phase shifters which have seen evidence development after the RF MEMS switch are brought forward as extension of RF switches itself. With broadband operating frequency ranges, evolving by minimizing the limitations of basic phase shifters possess. As DMTL phase shifter taken its prominence among other types, where the capacitive switch has major role in determining the performance in terms of speed. As techniques like pull-in voltage base techniques are least efficient in achieving the higher speeds, the mechanical tuning of the devices without altering the dimensions are preferred. In this paper a DMTL phase shifter that actuates with capacitive method is having modified beam structure to improve the frequency of the beam thus by enhancing the switching speed is adopted. With incorporation of the slits on the beam, switching speed is enhanced by $6-7 \%$, that operates at wide band applications from $18-40 \mathrm{GHz}$.
\end{abstract}

\section{Keywords}

MEMS; DMTL; Phase Shifters; RF MEMS Switch; Switching Time.

\section{Introduction}

The concept of Micro-machining technology that incorporates the science of Micro-electromechanical Systems (MEMS) and devices associated developed by Harvey C. Nathanson in 1965. This technology has gained its importance in all the fields of engineering and applications. Having the more advantageous with the traditional devices and systems, they possess the attributes of small in size, higher performance and better sensitivity and resolution making the MEMS more familiar in mechanical to biomedical industries. It gained the importance with Sensors and Actuators brought forward using this MEMS technology and various accelerometers are reported in literature [1-4]. This technology was brought to the applications of Radio frequency, since having the advantages of high performance in scaled down application, and in high frequency ranges. As in course with the upcoming of Millimeter wave base, the need for integration and scaling down the systems are essentials, the incorporation of MEMS technology is evident. This has given to the division of RF MEMS devices, being replacement for the traditional semiconductor based devices. In foremost RF MEMS switches are one, where extensive research and design modification have done so far since incorporation of Micro-machining was started in this field. As a result conventional PIN, MOSFET and GaAs based devices are replaced with RF MEMS switches.

Though the RF MEMS devices have advantages being the replacements, still have substantial challenges and setbacks when considering to operate at higher frequencies. RF passive devices like Phase shifters, couplers, splitters etc are quite more complicated with consideration of losses in terms of mechanical aspects rather than signal base loss mediums. As the MEMS deals with design that might consists of mechanical components, devices are prone to more loss concerned with vibrational and material base. One such device that has been in literature was RF MEMS base Phase Shifter which has wide application in RADAR and Wireless applications [5]. These devices are the considered to be two port devices, that are intended for the phase change from one port to another with loss less transmission of the signal. The conventional devices are designed with the help of PIN diodes, faced the challenges of low switching speed and design consideration. When these are replaced with the Field effect transistors, overcome the switching speed constraint but suffered with the transmission loss which is reported to be around 4-6 dB, and it is even higher with 8-9 dB for 12-18 GHz and $35 \mathrm{GHz}$ [6-10]. Few reported works have done the phase shifter with Barium titanate, that works with the Ferro-electric [11] are having the advantage of tuning but losses and cost are compromised.

In recent times, the RF passive components are adopted with the Micro-machining and calling down approaches with incorporation of the MEMS technology and the most adopted device with incorporation of MEMS technology was phase shifter. As the phase shifter using the MEMS technology is evolved with the RF MEMS switch design as the 
basic element. These RF MEMS switches are designed for various frequencies and with improved performance, by reducing the losses. This improvements brought the solution to challenges that traditional device are facing and cost of the devices are also brought down [11-13]. With this the Phase shifter as evolved with analog and digital base. Where Distributed Line (DTL), Loaded -line, reflection type and switched line are considered to be the categorized configurations of the shifter as per the design considerations. Among them DTL is also referred as the DMTL, most of the literature reported DMTL are analog within early 2000, with the introduction of digital communication and rapid development the urge for the digital base DMTL are very much needed. The main advantage with these, they are fabricated with most evident material silicon, ensuring the minimal cost and Losses at high frequency of operations [14]. But the challenges with this DMTL is having performance issues as the MEMS switches - basic building blocks of Shifter device having mechanical actuating element. This actuating element tend show instability at higher frequency of operations. There is always a compromise in speed and performance due this actuation element, as the larger the size more mechanical stability but less in frequency of actuation and vice versa. Another challenge lies with the fabrication of the devices at micro scale, which involves process other than silicon as well, even makes the things more worse.

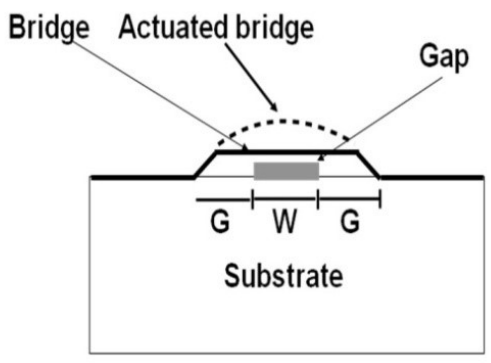

Fig. 1: RF MEMS Switch with Capacitive Actuation.

The present work is to emphasise on the mechanical stability improvement of the DMTL phase shifter which operates at the wide band from $18 \mathrm{GHz}$ to $40 \mathrm{GHz}$, using Metal- Insulator- Metal configuration. The performance and the stability are improved by the incorporation of Stress concentrated region on the beam of the RF MEMS switch which is basic element of the shifter. For improving faster switching, pull-in voltage, beam dimensional scaling and material alteration are mostly preferred in the literature, where the there is limitation of other parameters. But improving the device stability by stress concentration regions does alter the majority of devices considerations, but emphasis on the improvement of the frequency of the beam and thus switching speed is enhanced.

\section{DMTL Phase Shifter}

The design of the DMTL shifter is consider with the RF MEMS switch either in the configuration of Metal-Air-Metal (MAM) or Metal-Insulator-Metal (MIM) base configuration on a coplanar waveguide (CPW). Generally the material preferred for the CPW to place on a substrate is glass. Upon this CPW, a bridge configuration was constructed, which acts as simple RF MEMS switch that acts as the capacitive, since the bridge and the CPW plane acts as conductive plates. These two plates can be referred as the DC plate and RF connector plate and a gap between the two plates which acts the air gap for MEMS based capacitive switch. In between a dielectric was placed generally any oxide layer that acts as the insulator [14]. When an DC voltage is applied to the MEMS switch with a unified line, posing high resistance, the bridge breaks upon the application of the voltage and inline transmission of the wave is shifted. Generally any transmission line is expressed in terms of capacitance, inductance and resistance $\mathrm{Lt}, \mathrm{Ct}$ and $\mathrm{R}_{\mathrm{t}}$. This can represented with an electric circuit model in Fig 2.

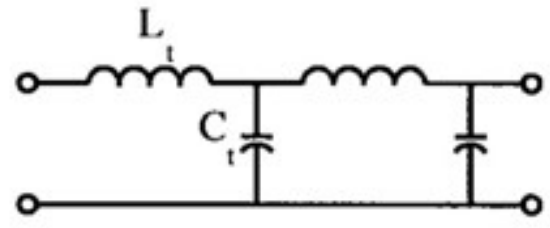

Fig. 2: Transmission Line in terms of Capacitance, Inductance and Resistance.

This entire configuration makes as RF MEMS switch configuration, so as for a phase shifter RF MEMS switch is basic block of it. When DC voltage applied to the Switch, the bridge is in down state that is actuated and when it was made to high resistance, simultaneously bridge becomes un actuated and opens up. In un actuated state only the AC capacitance is present and actuated state the charge built was discharged. In order to bias this switch also referred as 
pull-in voltage, a single high resistance line is used, this line was initially connected to one of the device and later moving it from switch to switch by bias line. This bias line has some in built resistance $\mathrm{R}_{\mathrm{b}}$. In most of the DMTL phase shifters, tuning of performance characteristics of the device are done using this $\mathrm{R}_{\mathrm{b}}$ and placing an inductor in series to this resistance by connecting to the RF connector plate where the bridge makes contact with it. This provides the discharge of the charge in more efficient way and any residual. This makes the bridge to be activated for charge in next cycle very much effectively. This tuning is expected to make the switching off the circuit faster by electric tuning of the device. But this technique has saturation with certain challenges, meeting the requirements with fabrication in certain time has limitation and other as bulking of the more inductance and capacitance might require higher pull in voltage making the design difficult for low power applications. Fig.3 explains the electric equivalent circuits of the single DMTL phase shifter under up state and down state. Further in this paper, the approach of mechanical tuning of the DMTL was made by applying the concept of the stress concentration regions. As the MEMS based RF switches are superior than the solid state devices, less pull-voltage, capable of handling high power and having lower spring constant are major challenges [15-17]. With adaptation of spring constant $(\mathrm{K})$ for the mechanical beam, which increases the actuate area of the switch electrodes and fine tuning the gap between the actuation electrodes, are concerning parameters to reduce the pull-in voltage. The stress controlled regions on the actuating beam will certainly help in reducing the up-state capacitance, that is major cause for insertion loss and in parallel with down state capacitance is modelled with high relative permittivity and thin film dielectric property for further increase to enhance the isolation property.

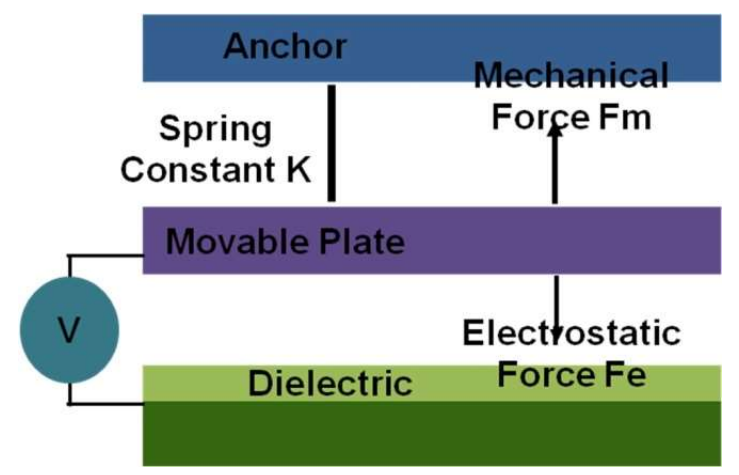

Fig. 3: Single DMTL Phase Shifter Under Up State and Down State.

\section{Proposed Wide-Band DMTL}

\section{A. Design and Structure of RF Switch}

It was mentioned in the section I, that phase shifters with MEMS technology are designed with the RF MEMS switch as the basic element. These was one way or other are alternative replacements for the solid state base devices. For this, it is concluded that the switch design was very important and prominent in modeling the Phase shifter at micro scales, involving the mechanical actuating elements. Challenges of the RF MEMS switch in terms of mechanical and the corelation of the mechanical aspects that alter the electric and capacitance parameters are discussed in Section II, are endorsed in this paper, as most of the RF MEMS switch design, the actuating element is considered to cantilever or beam. As both of them are subjected to the spring constant, up state capacitance are the more prone for alterations. All this, along with the fabrication challenges will question the performance and stability of the devices which are intended for use in wide application themes. As both of them are subjected to the spring constant, up state capacitance are the more prone for alterations. All this, along with the fabrication challenges will question the performance and stability of the devices which are intended for use in wide application themes. This is because, when the beam length is varied, that alters the CPW dimensions which changes the operating frequency and might increase the loss. The phase shift achieved, with these kind of alternation have the direct dependency on the operational wavelength (1/lambda) and effective length (L). As the DMTL is constructed by the serial loading of the transmission line (TL). This was further loaded with MEMS based massive components like inductors and capacitors. As these was in another way explained to be the RF capacitive Switch which has the actuating element as discussed earlier shown in Fig 2. The mechanical element that was proposed in this work is beam base. In such design the actuating element will be the top plate of the capacitor and the waveguide at the bottom being the ground plane.

It was observed the slow behaviour of the transmitted wave $(\mathrm{Vp}<<\mathrm{c})$, where $\mathrm{Vp}=$ phase velocity and $\mathrm{C}$ is velocity of light in vacuum. These are crucial parameters for the design of DMTL based phase shifter [18]. The capacitance is calibrated in these kinds of system with dynamic actuation of the actuating plate, which is done in plane deflection. This can be inferred as the parallel plate capacitor, but where one plate is having the dynamic nature. As these bridges are actuated by the external forces, and in case of the RF MEMS base passive components involving switches are 
actuated by the pull-in voltage. This is the subject that explains the upstate and down state of the device. Such device when constructed in series with unified CPW, makes things more complex and complicated in terms of performance etc. In such conditions the pull-in voltage in more complex parameters which needs to modeled. This work is approached with the wideband switch that is operated at $8-40 \mathrm{GHz}[3,19]$, that are reported in 1999, and further improved to make DMTL. As improvement of MEMS based micro-structures like single pole-double throw mechanics switches, that made to bring forward the digital base shifters. In most of the designs the microwave passive devices are preferred with the silicon and glass are major substrate materials and in case of the switch, thin film was deposited with silicon oxide, since the feasibility of fabrication processes. As switch consisting of the mechanical element, is subjected to stress and strain upon actuation and as switch is subjected numerous operations, stress and strain will tilt up with the hysteresis losses that won't be coming back to normal leaving behind, molecular disarrangement that creates the structural failure. So, the stress and strain with the different load conditions to be observed and time base analysis is must to understand the long terms functionality of the device and the Young's modulus was the deciding factor for this. Another challenging factor is switching speed. The switching speed depends upon the charge and discharge patterns of the capacitance that was built. But in case of MEMS switch, the frequency of the beam is responsible which is resonant frequency of the beam. It has the dependency on the pull-in voltage as well. As the residual charge that was left over also adds on to the next charge-discharge cycle.

The major functionality is considered with performance of the capacitive switch, which is intends upon the switching time $\left(t_{s}\right)$ - the time required from on/off. The switching time depends on the eq. 1 which is shown below

$$
T_{s}=\frac{3.67 * V p}{V s \omega}
$$

Where Vs is source voltage and which is incremented by 1.4 times of the actuation voltage in DMTL variety of base phase shifter using capacitive type switching. By altering this condition the above equation is further simplified to

$T_{s}=\frac{2.62}{\omega}$

Modifying the above equation the with the spring constant of the mechanical actuator

$$
T_{s}=2.67 \sqrt{m / k}
$$

This endorsed the implication regarding the frequency of switching action will depend on with respect to the length, width and thickness of the actuating element. In this paper, we have concentrated on the stress-controlled regions to un-alter the actuator lengths but to improve the frequency of switch that minimize the switching speed and further relates to fast switching[22].

\section{B. Functionality}

As it was discussed in above, the functionality of the phase shifter depends upon the switch operations. In this proposed device, switch is electrostatically actuated by using the capacitive MEMS switch, that is configured in shunt bias configuration. The actuating beam is placed on the top of CPW line. The capacitive switch is fixed on both sides of the of the CPW by separating it from the signal transmission line. In initial condition switch is up state and with the application of the voltage, it moves towards the dielectric state with deflection and this is referred as the down state. The switch is made simultaneously ON and OFF state with the this application of external voltage which are shown in the Fig 4.

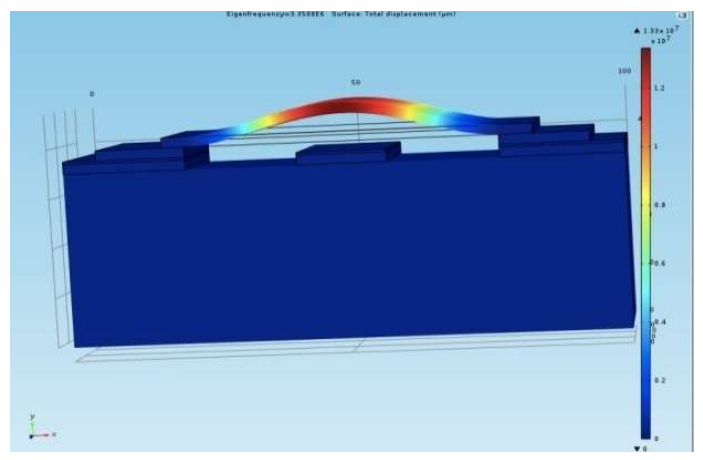

Fig. 4: RF MEMS Switch with Upstate. 


\section{Material Selection}

Material selection was crucial in terms of fabrication, design and performance of the device, in this paper the material selection was done with parametric analysis was done for understanding the material properties and their behaviour with respect to the design. The final material selection was done with the principle of Ashby's material selection process, that examples three basic guidelines for material selection for the device. According to Ashby's principle concept, embodiment and detail are major steps that governs the material selection. So as per this, the switch design depends upon the materials of the bring that defines the mechanical properties like spring constant, elasticity etc and dielectric material property that to built the charge. When these are chosen abruptly, chances of effecting the pull in voltage, switching speed and reliability of the devices are altered.

In order to choose the material for the beam and dielectric, a parametric sweep is carried out using Finite element Modeling (FEM) tool with different materials in consideration. An analysis between the Young's Modulus and thermal conductivity of different materials would certainly help in choosing the beam material for selection, in order to minimize the low pull in voltage. So that high conductive materials are compared in this analysis $(\mathrm{Au}, \mathrm{Pt}, \mathrm{Ni}, \mathrm{Cr}, \mathrm{Nb}$, $\mathrm{Al} 2 \mathrm{O} 3$ ) etc. as shows in Fig 6. And properties like insertion and isolation off RF passive components. It was mentioned in literature that, higher the Poisson ratio, the better capacitance is built between the dielectric and the beam. So, material having the higher Poisson's ratio are shown in the Table 1.

For the above table the, Silicon Nitride having the higher poisson's ratio, in most of the devices silicon oxide layer is preferred for its convenience of fabrication. But with techniques like Chemical Vapor Deposition techniques growing of silicon Nitride is also less complicated.

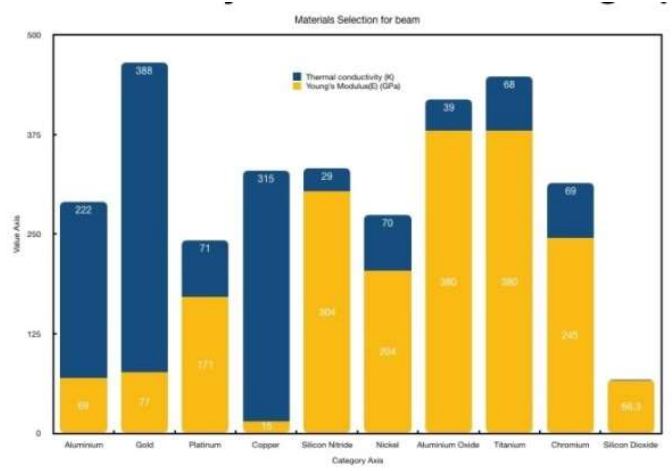

Fig. 5: Young's Modulus and Thermal Conductivity of Different Materials Table 1: Material Properties for Beam Consideration.

\begin{tabular}{|l|c|c|}
\hline \multirow{2}{*}{ Materials } & \multicolumn{2}{|c|}{ Properties } \\
\cline { 2 - 3 } & Young's Modulus(GPa) & Thermal Conductivity(K) \\
\hline Aluminium & 1521.91 & 1518.75 \\
\hline Gold & 2185.32 & 2183.67 \\
\hline Platinum & 3043.82 & 3042.12 \\
\hline Copper & 15 & 315 \\
\hline Silicon Nitride & 304 & 29 \\
\hline Nickel & 204 & 70 \\
\hline Aluminium Oxide & 380 & 39 \\
\hline Titanium & 380 & 68 \\
\hline Chromium & 245 & 69 \\
\hline Silicon Dioxide & 66.3 & 1.3 \\
\hline
\end{tabular}

\section{Stress Regions/Perforations}

In the proposed device of the DMTL, that uses the switch is incorporated with the perforation in order to improve the performance in terms of its switching speed which are shown in Fig 7. The perforation would help the device is minimizing the mass of the beam, which makes the frequency to be in higher state than usual calibrated resonant 
frequency. This will render the need of improving the switching speed and reduces the pull in voltage. But the challenges lies in making the perforation on the beam, that might alter the stability and reliability of the beam for longer durations. So a minimal perforation and maximum frequency shift is method that can be adopted to retain the stability and ensure frequency is in higher note. So regularization of the holes and balancing on the beam either side is important in tuning the frequency of the beam and it was stated that balanced perforation on top of the device would certainly improve the device frequency[23].

The main idea of this method is enhance the stress that to develop in the cantilever or beam and this technique does not involve any complex process of the micro-fabrication. With simple techniques by masking and etching, the perforation are framed. Initially this was reported for piezoresistive based MEMS sensor where sensitivity can be enhanced with larger amounts of stress [21]. In this paper, this approach was adopted to decrease the effective mass of the cantilever without altering the dimensions and shape of the beam. As from eq. 3 it can be stated that mass of the cantilever is inversely proportional to the frequency of the beam, where mass decreases the frequency increases. But too many perforation might increase the stress developed in the device that might alter the number switching of the device as it is involved with mechanical structures in it. In this design, as approach of the beam is utilized where the perforations are to balanced for beam being fixed at the both rear ends.

The perforations are made so in balanced state, either side of the beam to its centre and observed that frequency much better improved when the perforation are close to centre. As it was ensured that the set perforation are separated by equidistant, so that maximum deflection happens at the centre of the beam. The distance was measured to 4 micron and the each slit has 2 micron of each side (Square Shaped) and having $500 \mathrm{~nm}$ thickness as shown in Fig. 9. In such MEMS structure, we have observed principle mode frequency to be around $949 \mathrm{KHz}$.

\section{Pull-In-Voltage}

The approach of capacitive RF MEMS switch, one plate is fixed and the other plate is movable, with electrostatic actuation by the force created upon the application of the voltage between the top and bottom plates. With the help of restoration force $(\mathrm{Fm})$ and restoration force $(\mathrm{Fe})$, the motion of the plate is influenced.

$$
V_{p}=\sqrt{8 K / 27 \varepsilon \mathrm{Ag}^{3}}
$$

where $\mathrm{K}$ is spring constant, $\mathrm{E}$ is permittivity, $\mathrm{A}$ is area in millimeters and $\mathrm{g}$ is young's modulus.

\section{Simulation of the DMTL}

The RF capacitive MEMS switch, is designed with the above mentioned parameters and materials using the Finite Element Modeling (FEM) tools like COMSOL Multiphysics. In this mechanical behaviour and the resonant frequency of the switch are assessed and compared with and without SCR. Material sweep was performed to analyze the beam resonant frequency with variation to Young's Modulus of the device. The charge built during the up state was calibrated using the electrostatic AC/DC analysis in the tool by fixing the constraints and two parallel plates. For structural analysis the boundary conditions are ensured taken care of like fixing the beam on the rear ends for operating the beam in principle mode. The charge accumulated for an initial switch is around $70 \mathrm{pF}$ and each switch provides a shift of 6 degrees which regarded as the phase difference. With the shunt configuration of these capacitive switch bridges the designed phase shift can be achieved. In terms of Radio frequencies these kind of device acquire insertion loss and gain are the parameters that needs to considered. But along with that speed of the switch is other parameter that was discussed earlier. As the speed of the device is tuned with the pull-in voltage, but as the device consists of the mechanical structure, tuning mechanical would be more advantageous which might improve the overall performance of the device. So, with incorporation of the slit base devices, the main advantage is that beam frequency can be increased that alters the switching speed and this can be achieved without altering the dimensions of the devices. When the Eigen frequency analysis was carried for a single switch, has showed the frequency improvement by 59 $\mathrm{KHz}$ from $890 \mathrm{KHz}$ to $949 \mathrm{KHz}$ thus modified switch showing 6 to $7 \%$ improvement which is considerable to make modified DMTL operate much faster than the traditional DMTL. The main challenges that has to be considered in this design was to ensure that minimum strain was developed due to the slits on the beam, which might deform the device has to compromise overall durability of the device. 


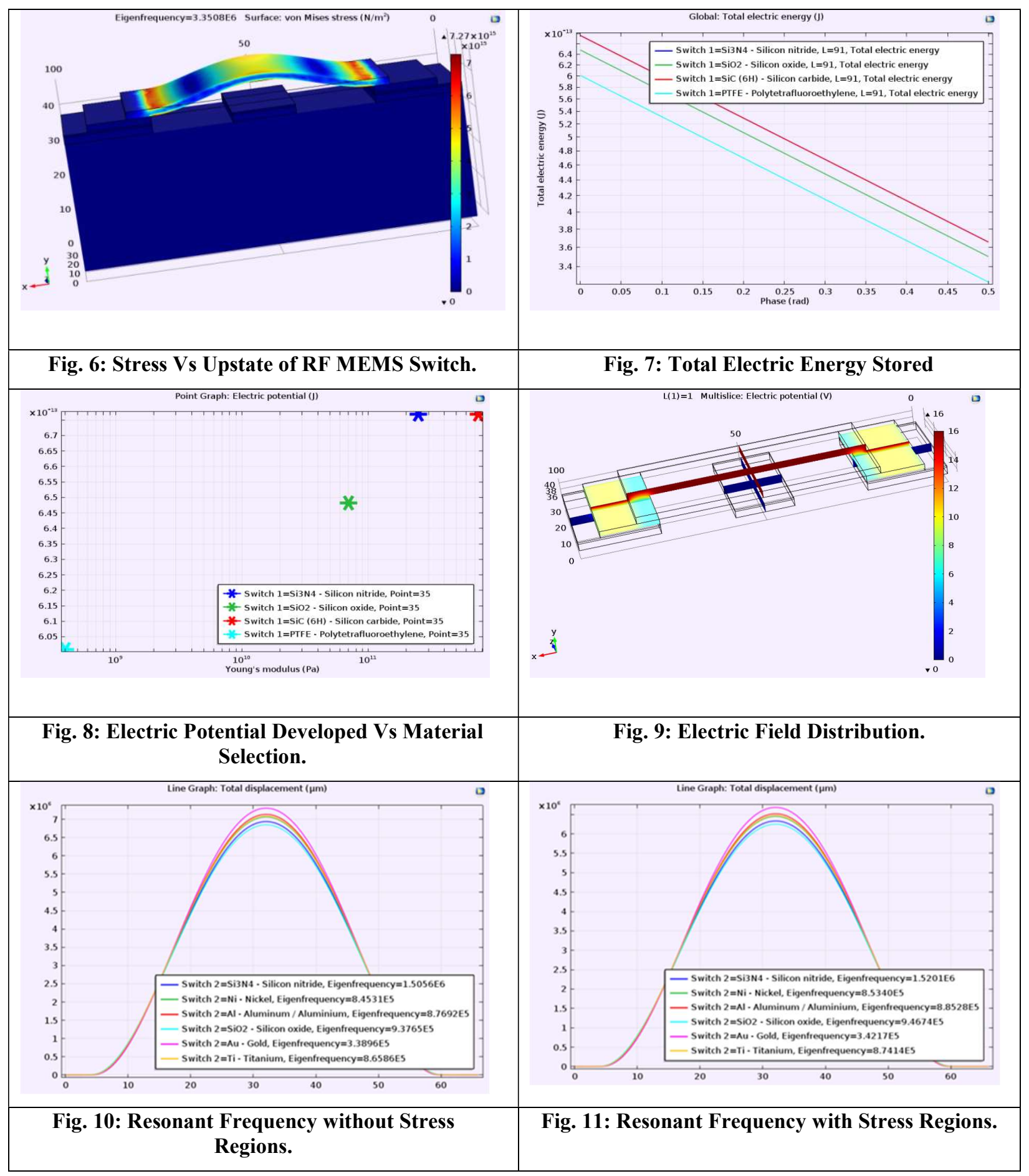



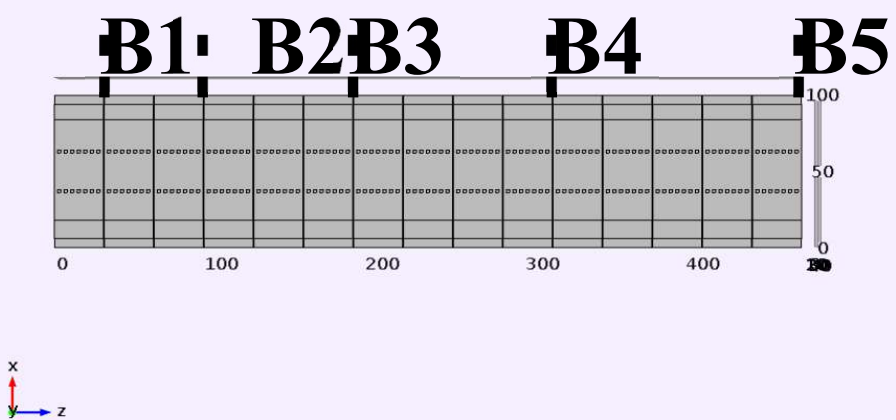

Fig. 12: 5-Bit DMTL Phase Shifter.

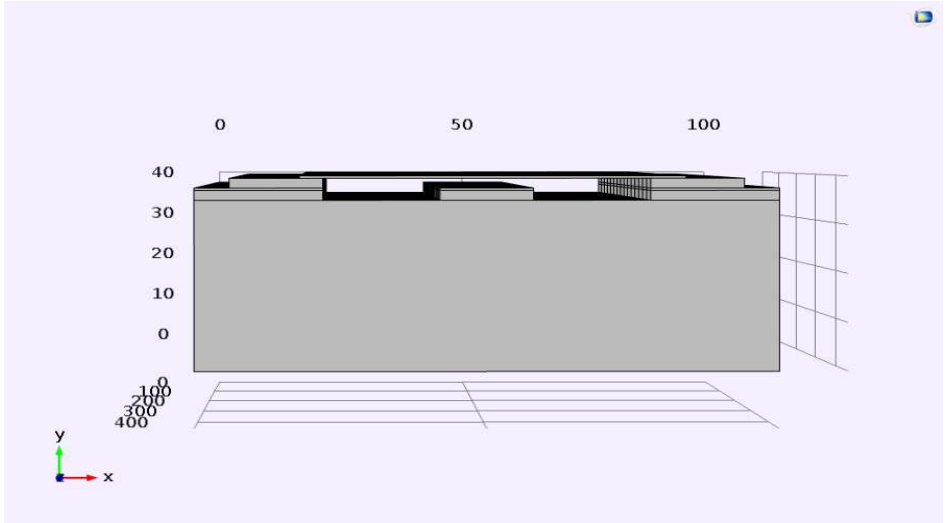

Fig. 13: Side View of Phase Shifter.

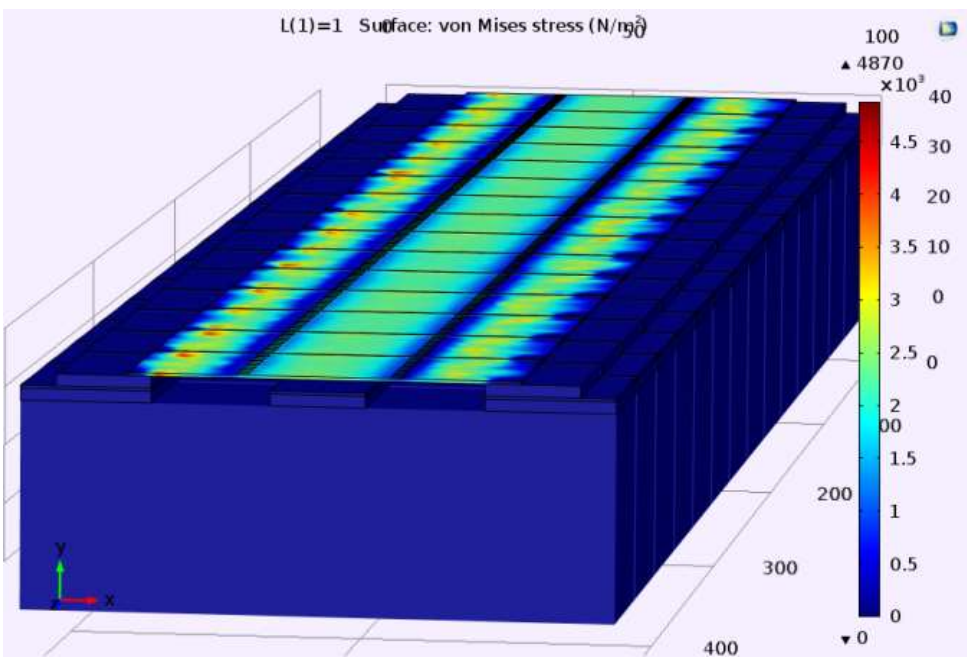

Fig. 14: Stress Developed Phase Shifter during Excitation. 


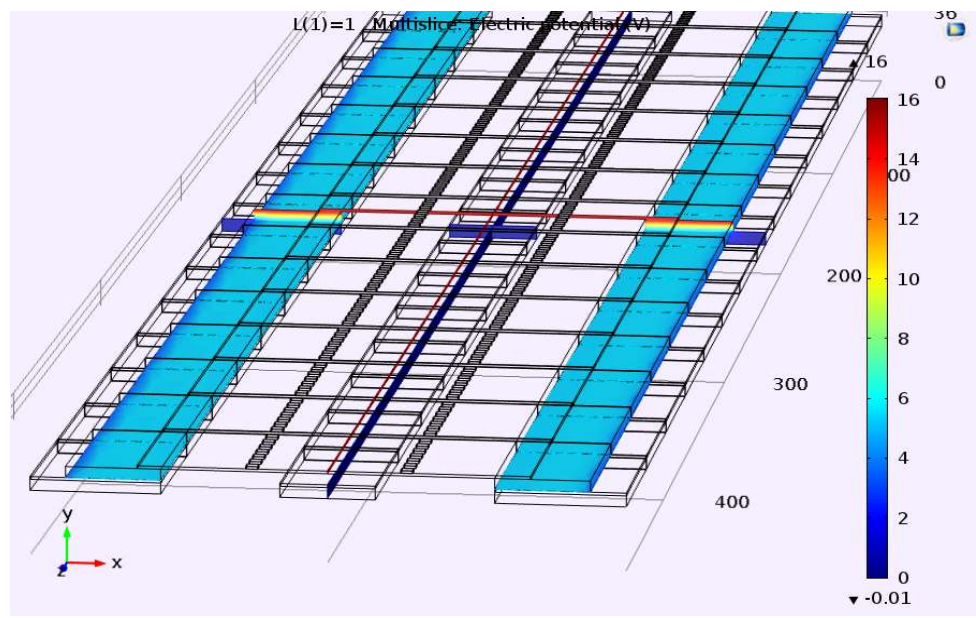

Fig. 15: Electric Field Distribution.

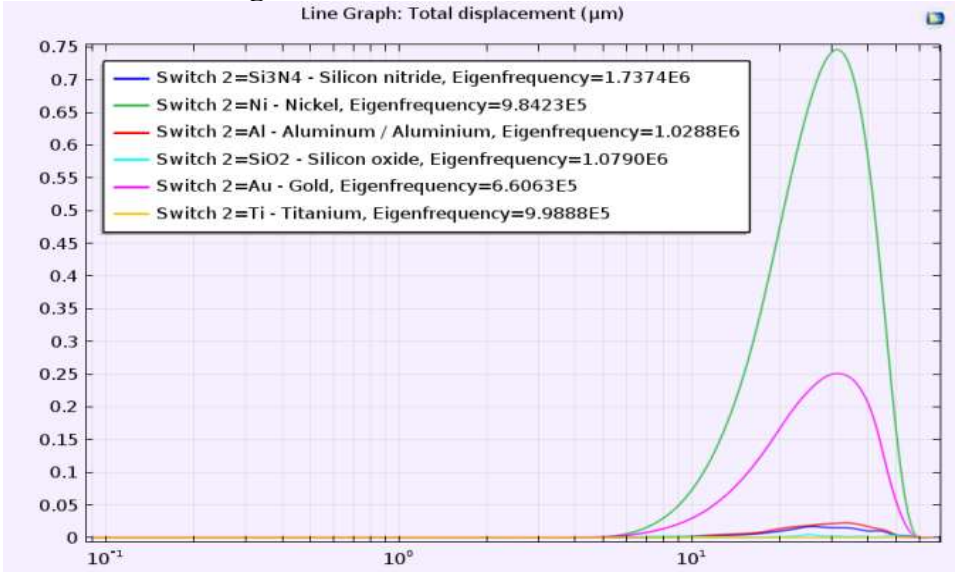

Fig. 16: Frequency Analysis of the Device.

\section{Conclusion}

The 5 bit DMTL phase shifter using modified switch that has built with stress concentration regions, it showed increase in the speed by resonant frequency changes of the beam and in parallel the pull voltage was also reduced. They was 8 $\%$ increase in switching speed of the device and pull in voltage was constraint to $12 \mathrm{~V}$. This paper has limited to the study of single bit calculation of the speed and has meet the overall speed and comparison with fabrication needs validated as future study.

\section{Acknowledgements}

This work is supported by the Pondicherry University, Puducherry to develop my academic and research knowledge by permitting me to do research work in the field of MEMS Phase shifters.

\section{References}

1. M. Makimoto and S. Yamashita, "Compact band-pass filters using stepped impedance resonators", IEEE Trans. Microwave Theory Tech., Vol. 40, pp. 16-19, Jan. 1979.

2. G.L. Mathieu, L. Young and E.M.T. Jones, Microwave Filters, Impedance-Matching Networks and Coupling Structures, $1^{\text {st }}$ ed., Artech House, Massachusetts, 1980.

3. S. Singh, "Design of microwave wideband impedance matching networks", M.S thesis, Dept. of Electrical Engineering, University of Nevada, Reno, USA, December 1995.

4. S.L. March, "Empirical formulas for the impedance and effective dielectric constant of covered microstrip for use in MIC CAD”, Proc. European Microwave Conf., Microwave Exhibitors \& Publishers Ltd., Kent, U.K., pp. 671-676, Sept. 1981.

5. H. Ogawa, "Technologies and standardization of milli meter-wave wireless personal area network", Digest of $10^{\text {th }}$ International Symposium on Microwave and Optical Technology”, Fukuoka, Japan, pp. 12-14, August 2225,2005 
6. O. Access, "We are Intec Open, the world's leading publisher of Open Access books Built by scientists, for scientists TOP $1 \%$ Based Sensor by Introducing Stress."

7. A. F. Recon, R. F. Mems, and P. Shifter, "A 15-40-GHz Frequency Reconfigurable RF MEMS Phase Shifter," vol. 61, no. 8, pp. 2865-2877, 2013.

8. Nguyen, C.T.-C.; Katehi, L.P.B.; Rebeiz, G.M. Micromachined Devices for wireless communications. Proc. IEEE 1998, 86, 1756-1768.

9. Yao, J.J. RF MEMS from a device perspective. J. Micromech. Microeng. 2000, 10, R9-R38.

10. K. Van Caekenberghe and T. Vähä-heikkilä, "An Analog RF MEMS Slotline True-Time-Delay Phase Shifter," pp. 1-9, 2008.

11. V. Nassereddine, "Milli meter-wave phase shifters based on tunable transmission lines in MEMS technology post-CMOS process To cite this version : HAL Id : tel-01492907 Déphaseurs en bande millimétrique basés sur des lignes à ondes lentes accordables en technologie MEMS dans un process post-CMOS .," 2018.

12. G. Mcfeetors and M. Okoniewski, "With Enhanced Tuning," vol. 16, no. 1, pp. 34-36, 2006.

13. B. Lakshminarayanan, T. M. Weller, S. Member, and A. Abstract, "Design and Modeling of 4-bit Slow-Wave MEMS Phase Shifters," vol. 54, no. 1, pp. 120-127, 2006.

14. B. Lacroix, A. Pothier, A. Crunteanu, and P. Blondy, "Phase Shifter Design Based on Fast RF MEMS Switched Capacitors," no. October, pp. 478-481, 2008.

15. S. Afrang and Æ. B. Yeop, "Distributed transmission line phase shifter using MEMS switches and inductors," pp. 1173-1183, 2008.

16. T. De Lauretis, A. Huyssen, and K. M. Woodward, "The Technological imagination : theories and fictions," Theor. Contemp. Cult. ; 3, no. 1, p. 201, 1980.

17. U. M. Spt, S. Switches, S. Dey, S. Member, and S. K. Koul, "Reliability Analysis of Ku-Band 5-bit Phase Shifters," vol. 63, no. 12, pp. 3997-4012, 2015.

18. C. R. Dietlein, S. Member, S. S. Bedair, J. S. Pulskamp, C. D. Meyer, and R. G. Polcawich, "Micro fabricated Thick-Cu PZT-MEMS Millimeter-Wave Topologies and Devices," vol. 25, no. 3, pp. 163-165, 2015.

19. S. Gong, S. Member, H. Shen, and N. S. Barker, "A 60-GHz 2-bit Switched-Line Phase Shifter Using SP4T RFMEMS Switches," vol. 59, no. 4, pp. 894-900, 2011.

20. N. Kingsley, S. Member, J. Papapolymerou, and S. Member, "Organic "Wafer-Scale ' Packaged Miniature," vol. 54, no. 3, pp. 1229-1236, 2006.

21. S. K. Koul and S. Dey, "K -Band 4-Bit Phase Shifter Using Two Back to Back MEMS SP16T Switching Networks," pp. 1-13, 2018.

22. K. S. Rao, C. H. G. Chand, and K. G. Sravani, "Design , Modeling and Analysis of Perforated RF MEMS Capacitive Shunt Switch,” IEEE Access, vol. 7, pp. 74869-74878, 2019.

23. E. Rufus and A. C. Actuation, "MEMS Capacitive Switch with Stress Concentration Regions for Wide band Applications Speed Applications," 2019 Int. Conf. Vis. Towar. Emerg. Trends Commun. Netw., pp. 1-5, 2019. 